\title{
Ergodicity of a Nonlinear Stochastic SIRS Epidemic Model with Regime-Switching Diffusions
}

\author{
Hongxia Liu, Juan Li, Mengnan Chi, Jinlei Liu, and Wencai Zhao \\ College of Mathematics and Systems Science, Shandong University of Science and Technology, Qingdao 266590, China \\ Correspondence should be addressed to Wencai Zhao; zhaowencai@sdust.edu.cn
}

Received 3 August 2020; Revised 23 September 2020; Accepted 3 October 2020; Published 23 October 2020

Academic Editor: Songbai Guo

Copyright (C) 2020 Hongxia Liu et al. This is an open access article distributed under the Creative Commons Attribution License, which permits unrestricted use, distribution, and reproduction in any medium, provided the original work is properly cited.

In this paper, taking both white noises and colored noises into consideration, a nonlinear stochastic SIRS epidemic model with regime switching is explored. The threshold parameter $R_{s}$ is found, and we investigate sufficient conditions for the existence of the ergodic stationary distribution of the positive solution. Finally, some numerical simulations are also carried out to demonstrate the analytical results.

\section{Introduction}

It is well known that the incidence rate plays a crucial role in studying the dynamics of infectious disease models. In general, bilinear incidence $\beta$ SI is considered in most infectious disease models [1, 2]. For example, Li and Ma [3] conducted the qualitative analyses of the SIS epidemic model with vaccination and varying total population size. Nakata and Kuniya [4] introduced the global dynamics of a class of SEIRS epidemic models in a periodic environment. In order to effectively investigate the rapid spread of the disease, it is rewarding to consider the behavioral changes and crowding effect of the infected individuals, as well as choose appropriate parameters to prevent the unbounded contact rate. Capasso and Serio [5] proposed the saturated incidence $(\beta \mathrm{SI} /(1+\alpha I))$, which is more reasonable than the bilinear incidence. For the detailed introduction of the saturated incidence, see [5].

The classical SIRS epidemic model with the saturated incidence rate is in the following form $[6,7]$ :

$$
\left\{\begin{array}{l}
\frac{\mathrm{d} S(t)}{\mathrm{d} t}=A-\frac{\beta S(t) I(t)}{1+\alpha I(t)}-\mu S(t)+\omega R(t), \\
\frac{\mathrm{d} I(t)}{\mathrm{d} t}=\frac{\beta S(t) I(t)}{1+\alpha I(t)}-(\mu+\lambda+d) I(t), \\
\frac{\mathrm{d} R(t)}{\mathrm{d} t}=\lambda I(t)-(\mu+\omega) R(t),
\end{array}\right.
$$

where $S(t), I(t)$, and $R(t)$ represent the number of susceptible, infected, and removed individuals at time $t$, respectively. $A$ denotes an input of new members into the population, $\beta$ stands for the transmission rate, $\mu$ is the natural mortality, $d$ is the death rate relative to the disease, $\lambda$ is the proportion of the infective class to the recovered class, and $\omega$ is the per capita rate of loss of immunity.

In nature, it is inevitable for a population to be affected by a variety of random factors $[8,9]$. Consequently, it is crucial to consider the randomness which might exist during the transmission of disease $[10,11]$. In general, there are two 
types of random perturbations to be considered in ecosystem modeling: one is white noise which can be described as Brownian motion [12-14], and the other is colored noise (also called telegraph noise) which can be described through a finite-state Markov chain [15-17]. In [15], Liu et al. investigated the threshold behavior of a multigroup SIRS epidemic model with standard incidence rates and Markovian switching. Lin and Jin [16] considered a stochastic
SIS epidemic model with regime switching; by verifying a Foster-Lyapunov condition, the threshold condition for the ergodicity is presented. Hu et al. [17] studied a stochastic SIS epidemic model with vaccination and nonlinear incidence under regime switching.

Motivated by the above literature, we study a nonlinear stochastic SIRS epidemic model with two kinds of random interference. The model is as follows:

$$
\left\{\begin{aligned}
\mathrm{d} S(t)= & \left((1-q(\xi(t))) A(\xi(t))-\frac{\beta(\xi(t)) S(t) I(t)}{1+\alpha I(t)}-\mu(\xi(t)) S(t)+\omega(\xi(t)) R(t)\right) \mathrm{d} t \\
& +\sigma_{1}(\xi(t)) S(t) \mathrm{d} B_{1}(t) \\
\mathrm{d} I(t)= & \left(\frac{\beta(\xi(t)) S(t) I(t)}{1+\alpha I(t)}-\mu(\xi(t)) I(t)-\lambda(\xi(t)) I(t)\right) \mathrm{d} t+\sigma_{2}(\xi(t)) I\left((t) \mathrm{d} B_{2}(t)\right. \\
\mathrm{d} R(t)= & (q(\xi(t)) A(\xi(t))+\lambda(\xi(t)) I(t)-\mu(\xi(t)) R(t)-\omega(\xi(t)) R(t)) \mathrm{d} t+\sigma_{3}(\xi(t)) R(t) \mathrm{d} B_{3}(t),
\end{aligned}\right.
$$

where $q$ is a fraction of vaccinated individuals for newborns. The incidence rate $\alpha$ contains the crowding effect of the infected individuals and should not be disturbed by the noises in the environment. $B_{i}(t)(i=1,2,3)$ denotes onedimensional standard Brownian motion, and $\sigma_{i}(i=1,2,3)$ is the intensity of white noise. $\xi(t)$ is a right-continuous Markov chain taking values in $\mathscr{U}=\{1,2, \ldots, m\}$, and the generator matrix of $\xi(t)$ is $\Gamma=\left(\gamma_{i j}\right)_{1 \leq i, j \leq m}$. The details of the Markov chain are presented in [18], which we omit here.

In this paper, the dynamic behaviors of stochastic differential system (2) are discussed. In Section 2, we get the conditions for the extinction and persistence in mean of the infected. In Section 3, we investigate the ergodicity of system (2) by constructing a suitable Lyapunov function. Finally, numerical simulations are given in Section 4 .

\section{The Extinction and Persistence of the Disease}

In system (2), let $N(t)=S(t)+I(t)+R(t)$; then, we have

$$
\begin{aligned}
\mathrm{d} N(t)= & {[A(\xi(t))-\mu(\xi(t)) N(t)] \mathrm{d} t+\sigma_{1}(\xi(t)) S(t) \mathrm{d} B_{1}(t) } \\
& +\sigma_{2}(\xi(t)) I(t) B_{2}(t) \\
& +\sigma_{3}(\xi(t)) R(t) \mathrm{d} B_{3}(t) .
\end{aligned}
$$

From Lemma 2.1 and Lemma 2.2 of [19], we have the following.

Lemma 1. For any initial value $(S(0), I(0), R(0)$, $r(0)) \in R_{+}^{3} \times \mathscr{M}$, the solution $(S(t), I(t), R(t))$ of system (2) has the following properties:

(1) $\lim _{t \rightarrow \infty}((S(t)+I(t)+R(t)) / t)=0$ a.s.

(2) $\lim _{t \rightarrow \infty}\left(\int_{0}^{t} S(u) d B_{1}(u) / t\right)=0, \quad \lim _{t \rightarrow \infty}\left(\int_{0}^{t} I(u) d\right.$ $\left.B_{2}(u) / t\right)=0, \lim _{t \rightarrow \infty}\left(\int_{0}^{t} R(u) d B_{3}(u) / t\right)=0$ a.s.

\section{Definition 1}

(1) If $\lim _{t \rightarrow \infty} I(t)=0$, then the disease tends to be extinct

(2) If $\lim _{t \rightarrow \infty} \inf (1 / t) E \int_{0}^{t} I(z) \mathrm{d} z>0$, then the disease tends to be persistent in mean

Define a matrix

$$
M=\left(\begin{array}{cccccc}
\mu(1)-\gamma_{11} & \cdots & -\gamma_{1 m} & 0 & \cdots & 0 \\
\vdots & & \vdots & \vdots & & \vdots \\
-\gamma_{m 1} & \cdots & \mu(m)-\gamma_{m m} & 0 & \cdots & 0 \\
-\omega(1) & \cdots & 0 & \mu(1)+\omega(1)-\gamma_{11} & \cdots & -\gamma_{1 m} \\
\vdots & & \vdots & \vdots & & \vdots \\
0 & \cdots & -\omega(m) & -\gamma_{m 1} & \cdots & \mu(m)+\omega(m)-\gamma_{m m}
\end{array}\right) .
$$


Following Lemma 1 in [20], we obtain that $M$ is a nonsingular $M$-matrix. By Theorem 2.10 (see Mao and Yuan $[21]), \quad$ for vector $\beta=(\beta(1), \beta(2), \ldots, \beta(m), 0,0, \ldots$, $0)^{T} \in R^{2 m}$, the equation $M \eta=\beta$ has a unique solution:

$$
\eta=\left(d_{1}(1), d_{1}(2), \ldots, d_{1}(m), d_{2}(1), d_{2}(2), \ldots, d_{2}(m)\right)^{T} \in R_{+}^{2 m} .
$$

That is,

$$
\left\{\begin{array}{l}
\beta(k)-d_{1}(k) \mu(k)+\sum_{l \in M} \gamma_{k l} \mathrm{~d}_{1}(l)=0, \quad k=1,2, \ldots, m \\
d_{1}(k) \omega(k)-d_{2}(k)(\mu(k)+\omega(k))+\sum_{l \in M} \gamma_{k l} \mathrm{~d}_{2}(l)=0, \quad k=1,2, \ldots, m .
\end{array}\right.
$$

Then, we define a parameter

$$
R_{s}=\Sigma_{k \in \mathscr{M}} \pi_{k}\left[d_{1}(k)(1-q(k)) A(k)+d_{2}(k) q(k) A(k)-\left(\mu(k)+\lambda(k)+\frac{\sigma_{2}^{2}(k)}{2}\right)\right] .
$$

Theorem 1. For any initial value $(S(0), I(0), R(0)$, $r(0)) \in R_{+}^{3} \times \mathscr{M}$, assume that $\alpha+\widehat{d}_{1}-d_{2}>0$; then,

(1) The disease $I(t)$ of system (2) is extinct when $R_{s}<0$

(2) The disease $I(t)$ of system (2) is persistent in mean when $R_{s}>0$

Proof. Define the function

$U(S(t), I(t), R(t), k)=U_{1}(S(t), I(t), R(t), k)+U_{2}(I(t))$, where

$$
\begin{aligned}
U_{1}(S(t), I(t), R(t), k) & =d_{1}(k)(S(t)+I(t))+d_{2}(k) R(t), \\
U_{2}(I(t)) & =\ln I(t)+\alpha I(t) .
\end{aligned}
$$

Then, applying Itô's formula, we have

$$
\begin{aligned}
L U_{1}= & d_{1}(k)[(1-q(k)) A(k)-\mu(k) S(t)-(\mu(k)+\lambda(k)) I(t)+\omega(k) R(t)] \\
& +d_{2}(k)[q(k) A(k)+\lambda(k) I(t)-(\mu(k)+\omega(k)) R(t)]+(S(t)+I(t)) \sum_{l=1}^{m} \gamma_{k l} \mathrm{~d}_{1}(l)+R(t) \sum_{l=1}^{m} \gamma_{k l} \mathrm{~d}_{2}(l) \\
= & d_{1}(k)(1-q(k)) A(k)+d_{2}(k) q(k) A(k)+\left[-d_{1}(k) \mu(k)+\sum_{l=1}^{m} \gamma_{k l} \mathrm{~d}_{1}(l)\right] S(t) \\
& -\left[(\mu(k)+\lambda(k)) d_{1}(k)-\lambda(k) d_{2}(k)-\sum_{l=1}^{m} \gamma_{k l} \mathrm{~d}_{1}(l)\right] I(t) \\
& +\left[d_{1}(k) \omega(k)-d_{2}(k)(\mu(k)+\omega(k))+\sum_{l=1}^{m} \gamma_{k l} \mathrm{~d}_{2}(l)\right] R(t) \\
= & d_{1}(k)(1-q(k)) A(k)+d_{2}(k) q(k) A(k)-\beta(k) S(t)-\left[\beta(k)+\lambda(k) d_{1}(k)-\lambda(k) d_{2}(k)\right] I(t), \\
L U_{2}= & \frac{\beta(k) S(t)}{1+\alpha I(t)}-\left(\mu(k)+\lambda(k)+\frac{\sigma_{2}^{2}(k)}{2}\right)+\frac{\alpha \beta(k) S(t) I(t)}{1+\alpha I(t)}-\alpha(\mu(k)+\lambda(k)) I(t) \\
= & \beta(k) S(t)-\alpha(\mu(k)+\lambda(k)) I(t)-\left(\mu(k)+\lambda(k)+\frac{\sigma_{2}^{2}(k)}{2}\right) .
\end{aligned}
$$


By (10) and (11), one can obtain that

$$
\begin{aligned}
L U= & d_{1}(k)(1-q(k)) A(k)+d_{2}(k) q(k) A(k)-\left(\mu(k)+\lambda(k)+\frac{\sigma_{2}^{2}(k)}{2}\right) \\
& -\left[\beta(k)+\lambda(k) d_{1}(k)-\lambda(k) d_{2}(k)+\alpha \mu(k)+\alpha \lambda(k)\right] I(t) \\
= & d_{1}(k)(1-q(k)) A(k)+d_{2}(k) q(k) A(k)-\left(\mu(k)+\lambda(k)+\frac{\sigma_{2}^{2}(k)}{2}\right) \\
& -\left[\beta(k)+\lambda(k)\left(\alpha+d_{1}(k)-d_{2}(k)\right)+\alpha \mu(k)\right] I(t) .
\end{aligned}
$$

An application of generalized Itô's formula yields

$$
\begin{aligned}
& U(S(t), I(t), R(t), \xi(t)) \\
& =U(S(0), I(0), R(0), \xi(0))+\int_{0}^{t} L U(S(z), I(z), R(z), \xi(z)) \mathrm{d} z+\int_{0}^{t} d_{1}(\xi(z)) \sigma_{1}(\xi(z)) S(z) \mathrm{d} B_{1}(z) \\
& \quad+\int_{0}^{t}\left[1+\left(\alpha+d_{1}(\xi(z))\right) I(z)\right] \sigma_{2}(\xi(z)) \mathrm{d} B_{2}(z)+\int_{0}^{t} d_{2}(\xi(z)) \sigma_{3}(\xi(z)) R(z) \mathrm{d} B_{3}(z) \\
& \quad+\int_{0}^{t} \int_{R}\left(U\left(S(z), I(z), R(z), i_{0}+\ell(\xi(z), l)\right)\right)-U(S(z), I(z), R(z), \xi(z)) \mu(d z, d l)
\end{aligned}
$$

where $\mu(\mathrm{d} z, \mathrm{~d} l)=\gamma(\mathrm{d} z, \mathrm{~d} l)-\mu(\mathrm{d} l) \mathrm{d} z$ is a martingale measure. By using Lemma 1.9 of [21], we can get

$$
E[U(S(t), I(t), R(t), \xi(t))]=E[U(S(0), I(0), R(0), \xi(0))]+E\left[\int_{0}^{t} L U(S(z), I(z), R(z), \xi(z)) \mathrm{d} z\right]
$$

Then,

$$
\begin{aligned}
& E\left[\frac{U(S(t), I(t), R(t), \xi(t))]}{t}\right] \\
& =E\left[\frac{U(S(0), I(0), R(0), \xi(0))}{t}\right] \\
& \quad+E\left\{\frac{1}{t} \int_{0}^{t}\left[d_{1}(\xi(z))(1-q(\xi(z))) A(\xi(z))+d_{2}(\xi(z)) q(\xi(z)) A(\xi(z))-\left(\mu(\xi(z))+\lambda(\xi(z))+\frac{\sigma_{2}^{2}(\xi(z))}{2}\right) \mathrm{d} z\right\}\right. \\
& \quad-E\left\{\frac{1}{t} \int_{0}^{t}\left[\beta(\xi(z))+\lambda(\xi(z))\left(\alpha+d_{1}(\xi(z))-d_{2}(\xi(z))\right)+\alpha \mu(\xi(z))\right] I(z) \mathrm{d} z\right\} .
\end{aligned}
$$


Hence,

$$
\begin{aligned}
\frac{R_{s}}{\widetilde{\beta}+\breve{\lambda}\left(\alpha+\breve{d}_{1}-\widehat{d}_{2}\right)+\alpha \breve{\mu}} & \leq \lim _{t \longrightarrow \infty} \inf \frac{1}{t} E \int_{0}^{t} I(z) \mathrm{d} z \\
& \leq \lim _{t \rightarrow \infty} \sup \frac{1}{t} E \int_{0}^{t} I(z) \mathrm{d} z \\
& \leq \frac{R_{s}}{\widehat{\beta}+\widehat{\lambda}\left(\alpha+\widehat{d}_{1}-\breve{d}_{2}\right)+\alpha \widehat{\mu}}
\end{aligned}
$$

In conclusion, based on the condition $\alpha+\widehat{d}_{1}-\breve{d}_{2}>0$, we have the following:

(1) If $R_{s}<0$, then $\lim _{t \rightarrow \infty} I(t)=0$, a.s.

(2) If $R_{s}>0$, then $\lim _{t \rightarrow \infty} \inf (1 / t) E \int_{0}^{t} I(z) \mathrm{d} z \geq\left(R_{s} / \breve{\beta}+\right.$ $\left.\lambda\left(\alpha+d_{1}-\widehat{d}_{2}\right)+\alpha \breve{\mu}\right)>0$, and the disease $I(t)$ of system (2) is persistent in mean
Remark 1. According to Theorem 1, if the intensity of white noise is large enough that the condition $R_{s}<0$ holds, then the disease dies out with probability 1 . Conversely, if $R_{s}>0$, the disease of system (2) is persistent in mean. This means that the presence of environmental noise is conducive to disease control.

\section{Ergodic Stationary Distribution of System (2)}

The study of the ergodicity and stationary distribution has been widely concerned by many scholars [22, 23]. In this section, in order to investigate the ergodic property of system (2), we establish a suitable Lyapunov function with Markov conversion.

Theorem 2. For any initial value $(S(0), I(0), R(0)$, $\xi(0)) \in R_{+}^{3} \times \mathscr{M}$, if $R_{s}>0$, stochastic process $(S(t), I(t)$, $R(t), \xi(t))$ of system (2) is ergodic and has a unique stationary distribution in $R_{+}^{3} \times \mathscr{M}$.

Proof. Let $x(t)=\ln S(t), y(t)=\ln I(t)$, and $z(t)=\ln R(t)$. System (2) can be transformed into

$$
\left\{\begin{array}{l}
\mathrm{d} x(t)=\left[\frac{(1-q(\xi(t))) A(\xi(t))}{e^{x(t)}}-\frac{\beta(\xi(t)) e^{y(t)}}{1+\alpha e^{y(t)}}-\mu(\xi(t))+\frac{\omega(\xi(t)) e^{z(t)}}{e^{x(t)}}-\frac{1}{2} \sigma_{1}^{2}(\xi(t))\right] \mathrm{d} t+\sigma_{1}(\xi(t)) \mathrm{d} B_{1}(t), \\
\mathrm{d} y(t)=\left[\frac{\beta(\xi(t)) e^{x(t)}}{1+\alpha e^{y(t)}}-\mu(\xi(t))-\lambda(\xi(t))-\frac{1}{2} \sigma_{2}^{2}(\xi(t))\right] \mathrm{d} t+\sigma_{2}(\xi(t)) \mathrm{d} B_{2}(t), \\
\mathrm{d} z(t)=\left[\frac{q(\xi(t)) A(\xi(t))}{e^{z(t)}}+\frac{\lambda(\xi(t)) e^{y(t)}}{e^{z(t)}}-\mu(\xi(t))-\omega(\xi(t))-\frac{1}{2} \sigma_{3}^{2}(\xi(t))\right] \mathrm{d} t+\sigma_{3}(\xi(t)) \mathrm{d} B_{3}(t) .
\end{array}\right.
$$

The ergodic property of system (2) is the same as that of system (17). We need to prove that system (17) satisfies the three conditions of Lemma 2.2 in [18]. Obviously, conditions
(1) and (2) are satisfied. Hence, we only need to prove condition (3).

Define

$$
H(x, y, z, k)=\frac{1}{\vartheta+1}\left(e^{x}+e^{y}+e^{z}\right)^{\vartheta+1}-a\left[d_{1}(k)\left(e^{x}+e^{y}\right)+d_{2}(k) e^{z}+y+\alpha e^{y}+\tau(k)\right]-x-z,
$$

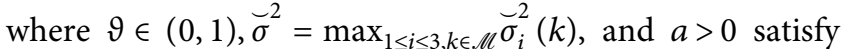
$\widehat{\mu}-(\vartheta / 2) \breve{\sigma}^{2}>0,-a R_{s}+2 \breve{\mu}+\breve{\omega}+(1 / 2) \breve{\sigma}_{1}^{2}+(1 / 2) \breve{\sigma}_{3}^{2} \leq-2 . \tau_{k}$ will be determined later. There exists a unique point $\left(x_{0}, y_{0}, z_{0}, k\right)$ which is the minimum value of $H(x, y, z, k)$. Set

$$
V(x, y, z, k)=H(x, y, z, k)-H\left(x_{0}, y_{0}, z_{0}, k\right) .
$$

Denote

$$
\begin{aligned}
& V_{1}=\frac{1}{\vartheta+1}\left(e^{x}+e^{y}+e^{z}\right)^{9+1}, \\
& V_{2}=-\left[d_{1}(k)\left(e^{x}+e^{y}\right)+d_{2}(k) e^{z}+y+\alpha e^{y}+\tau(k)\right], \\
& V_{3}=-x, \\
& V_{4}=-z .
\end{aligned}
$$

An application of generalized Itô's formula yields 


$$
\begin{aligned}
& L V_{1}=\left(e^{x}+e^{y}+e^{z}\right)^{\vartheta}\left(A(k)-\mu(k) e^{x}-\mu(k) e^{y}-\mu(k) e^{z}\right) \\
& +\frac{9}{2}\left(e^{x}+e^{y}+e^{z}\right)^{9-1}\left(\sigma_{1}^{2}(k) e^{2 x}+\sigma_{2}^{2}(k) e^{2 y}+\sigma_{3}^{2}(k) e^{2 z}\right) \\
& \leq 3^{\vartheta} A(k)\left(e^{\vartheta x}+e^{\vartheta y}+e^{\vartheta z}\right)-\mu(k) e^{(\vartheta+1) x}-\mu(k) e^{(\vartheta+1) y}-\mu(k) e^{(\vartheta+1) z} \\
& +\frac{\vartheta}{2} \sigma_{1}^{2}(k) e^{(\vartheta+1) x}+\frac{\vartheta}{2} \sigma_{2}^{2}(k) e^{(\vartheta+1) y}+\frac{\vartheta}{2} \sigma_{3}^{2}(k) e^{(\vartheta+1) z} \\
& =-\left(\mu(k)-\frac{\vartheta}{2} \sigma_{1}^{2}(k)\right) e^{(\vartheta+1) x}-\left(\mu(k)-\frac{\vartheta}{2} \sigma_{2}^{2}(k)\right) e^{(\vartheta+1) y} \\
& -\left(\mu(k)-\frac{\vartheta}{2} \sigma_{3}^{2}(k)\right) e^{(\vartheta+1) z}+3^{\vartheta} A(k)\left(e^{\vartheta x}+e^{\vartheta y}+e^{\vartheta z}\right)
\end{aligned}
$$

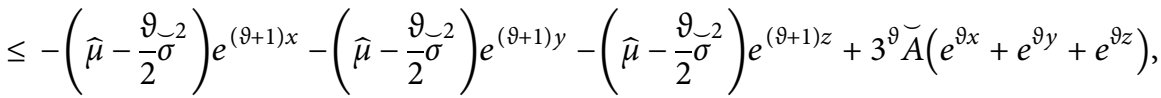

$$
\begin{aligned}
& L V_{2}=-d_{1}(k)(1-q(k)) A(k)-d_{2}(k) q(k) A(k)+\mu(k)+\lambda(k)+\frac{\sigma_{2}^{2}(k)}{2}-\sum_{l=1}^{m} \gamma_{k l} \tau(l) \\
& +e^{x}\left[d_{1}(k) \mu(k)-\beta(k)-\sum_{l \in \mathscr{M}} \gamma_{k l} \mathrm{~d}_{1}(l)\right]+e^{y}\left[\left(d_{1}(k)+\alpha\right)(\mu(k)+\lambda(k))-d_{2}(k) \lambda(k)-\sum_{l \in \mathscr{M}} \gamma_{k l} \mathrm{~d}_{1}(l)\right] \\
& +e^{z}\left[-d_{1}(k) \omega(k)+d_{2}(k)(\mu(k)+\omega(k))-\sum_{l \in \mathscr{M}} \gamma_{k l} \mathrm{~d}_{2}(l)\right] \\
& =-G_{0 k}-\sum_{l=1}^{m} \gamma_{k l} \tau(l)+e^{y}\left[\beta(k)+\left(d_{1}(k)-d_{2}(k)\right) \lambda(k)+\alpha(\mu(k)+\lambda(k))\right] \text {, }
\end{aligned}
$$

where we used equation (4) and

$G_{0 k}=d_{1}(k)(1-q(k)) A(k)+d_{2}(k) q(k) A(k)-\mu(k)-\lambda(k)-\frac{\sigma_{2}^{2}(k)}{2}$.

Let $\tau=\left(\tau_{1}, \ldots, \tau_{m}\right)^{T}$ be the solution of the following Poisson system:

$$
\Gamma \tau=\left(\sum_{k=1}^{m} \pi_{k} G_{0 k}\right) \overrightarrow{1}-\left(\begin{array}{c}
G_{01} \\
\vdots \\
G_{0 m}
\end{array}\right),
$$

where $G=\left(G_{01}, G_{02}, \ldots, G_{0 m}\right)^{T}$.

Substituting this equality into (22), we obtain

$$
\begin{aligned}
& L V_{2}=-\sum_{k=1}^{m} \pi_{k} G_{0 k}+e^{y}\left[\beta(k)+\left(d_{1}(k)-d_{2}(k)\right) \lambda(k)+\alpha(\mu(k)+\lambda(k))\right] \\
& L V_{3}=-\left(\frac{(1-q(k)) A(k)}{e^{x}}-\frac{\beta(k) e^{y}}{1+\alpha e^{y}}+\frac{\omega(k) e^{z}}{e^{x}}-\mu(k)-\frac{1}{2} \sigma_{1}^{2}(k)\right), \\
& L V_{4}=-\frac{q(k) A(k)}{e^{z}}-\frac{\lambda(k) e^{y}}{e^{z}}+\left(\mu(k)+\omega(k)+\frac{1}{2} \sigma_{3}^{2}(k)\right) .
\end{aligned}
$$




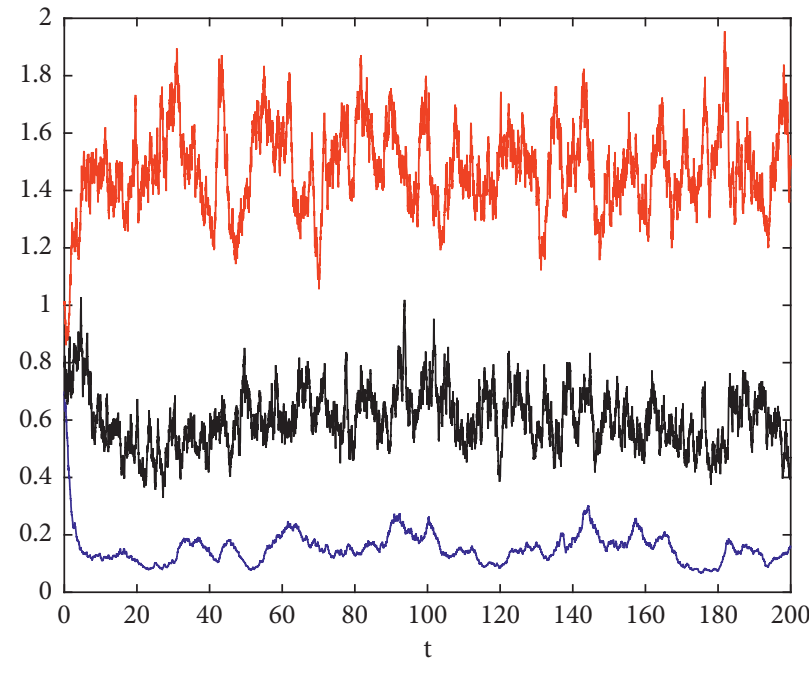

$k=1, \sigma_{2}(1)=0.05$

$-S$
$-I$
$-R$

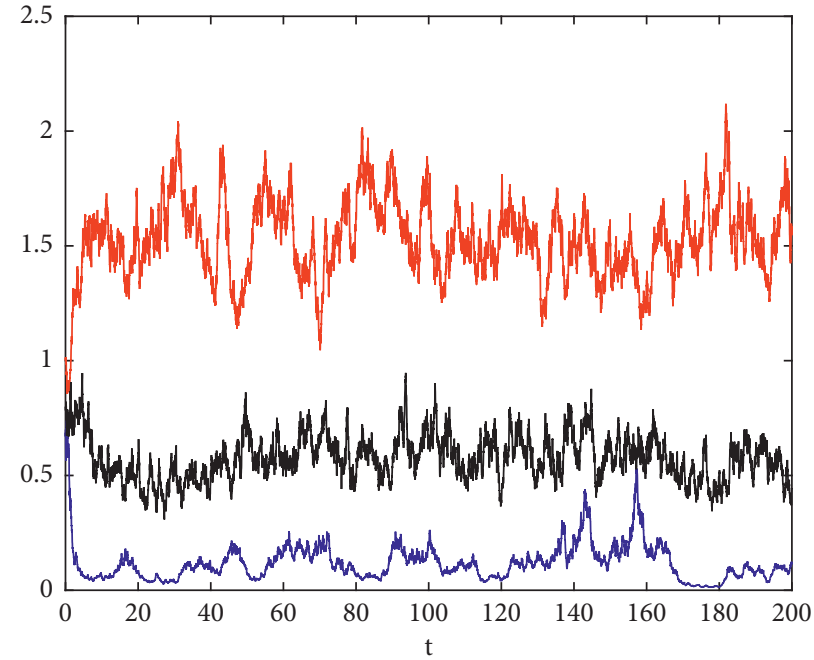

$k=2, \sigma_{2}(2)=0.1$

$-S$
$-I$
-

(a)

(b)

Figure 1: Simulations of the solution $(S(t), I(t), R(t))$ to stochastic model $(2)$ with $(S(0), I(0), R(0))=(1,0.7,0.8) .(\mathrm{a}) k=1, \sigma_{2}(1)=0.05$. (b) $k=2, \sigma_{2}(2)=0.1$.

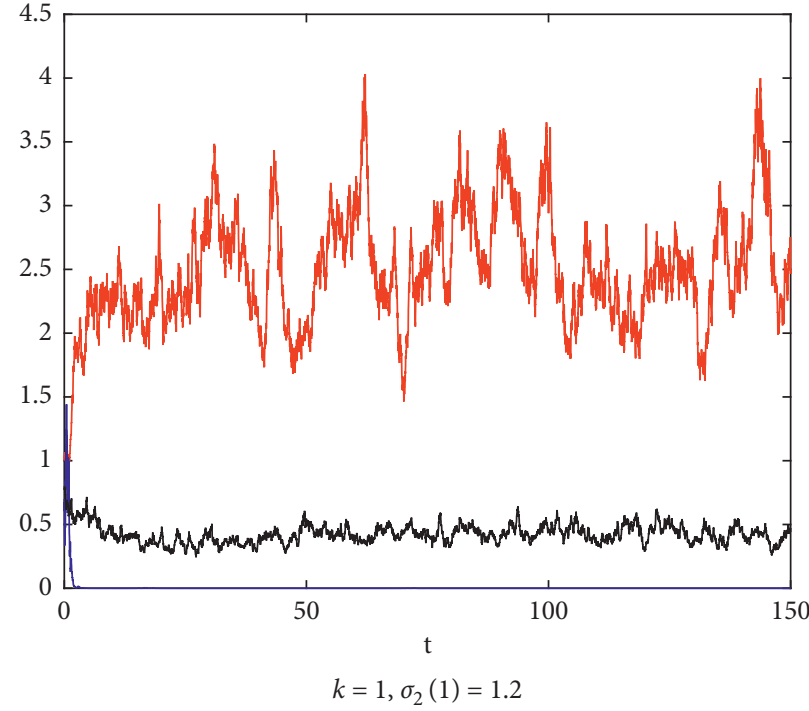

$-S$
$-I$
$-R$

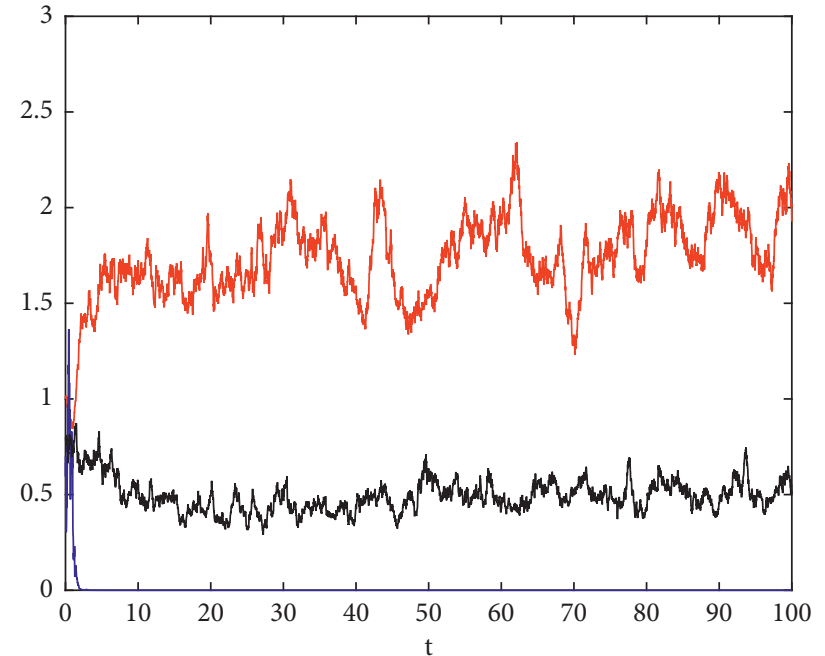

$k=2, \sigma_{2}(2)=1.2$

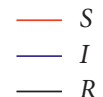

(a)

(b)

Figure 2: Simulations of the solution $(S(t), I(t), R(t))$ to stochastic model (2) with $(S(0), I(0), R(0))=(1,0.7,0.8) .(\mathrm{a}) k=1, \sigma_{2}(1)=1.2$. (b) $k=2, \sigma_{2}(2)=1.2$. 

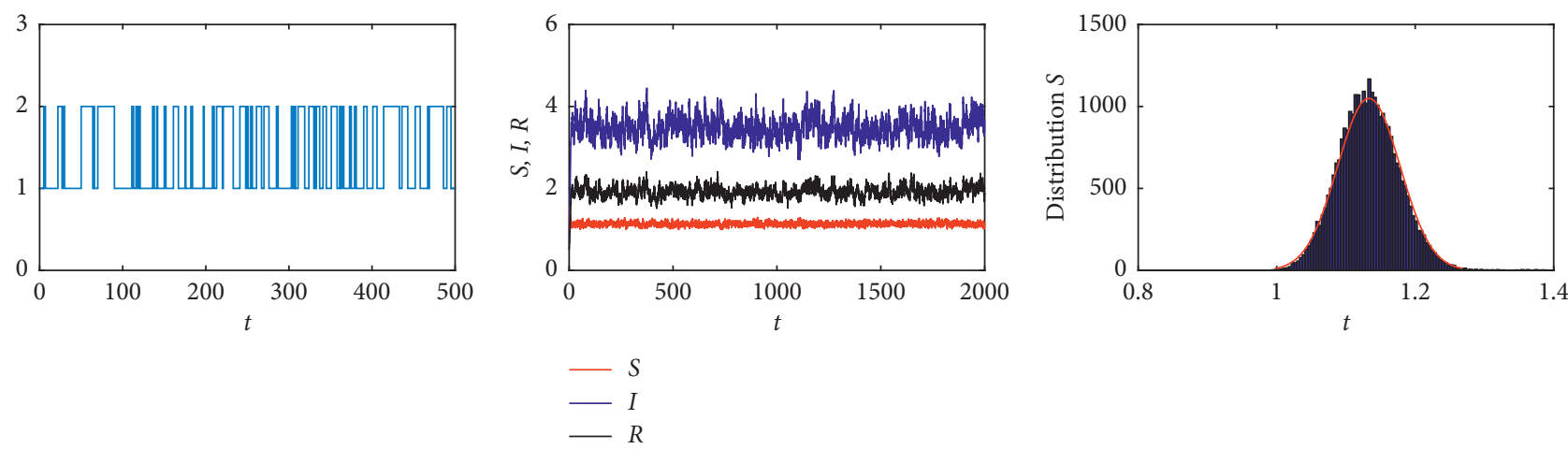

(a)

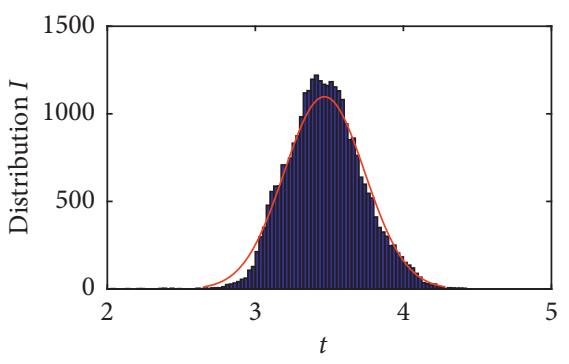

(d) (b)

(c)

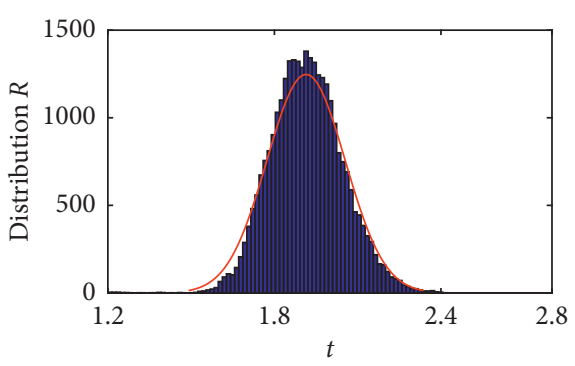

(e)

Figure 3: (a) Markov chain. (b), (c), (d), and (e) Distributions of $S(t), I(t)$, and $R(t)$ of model (2).

Combining (21) and (25)-(27), we have

$$
\begin{aligned}
L V(x, y, z, k)= & L V_{1}+a L V_{2}+L V_{3}+L V_{4} \\
\leq & -\left(\widehat{\mu}-\frac{\vartheta}{2} \breve{\sigma}^{2}\right) e^{(\vartheta+1) x}+3^{\vartheta} \breve{A} e^{\vartheta x}-\frac{(1-\breve{q}) \widehat{A}}{e^{x}}-\left(\widehat{\mu}-\frac{\vartheta}{2} \breve{\sigma}^{2}\right) e^{(\vartheta+1) y} \\
& +3^{\vartheta} \breve{A} e^{9 y}+a e^{y}\left[\breve{\beta}+\breve{\lambda}\left(\breve{d}_{1}-\widehat{d}_{2}\right)+\alpha(\breve{\mu}+\breve{\lambda})\right]+\breve{\beta} e^{y} \\
& -\left(\widehat{\mu}-\frac{\vartheta}{2} \breve{\sigma}^{2}\right) e^{(\vartheta+1) z}+3^{\vartheta} \breve{A} e^{9 z}-\frac{\widehat{q} \widehat{A}}{e^{z}} \\
& -a R_{s}+2 \breve{\mu}+\breve{\omega}+\frac{1}{2} \breve{\sigma}_{1}^{2}+\frac{1}{2} \breve{\sigma}_{3}^{2} .
\end{aligned}
$$

Consequently, take $\rho$ sufficiently large, and let $U=$ $(-\rho, \rho) \times(-\rho, \rho) \times(-\rho, \rho)$; then, we have $L V(x, y, z, k) \leq-$ 1 for any $(x, y, z, k) \in U^{c} \times \mathscr{M}$. According to Lemma 2.2 in [18], we know that $(x(t), y(t), z(t), \xi(t))$ is ergodic and positive recurrent.

This proof is completed.

\section{Conclusions and Numerical Simulations}

This paper investigated a nonlinear epidemic disease model with two kinds of noise disturbances. The threshold of extinction and persistence in mean is obtained. (i) If $R_{s}<0$, the infected individuals tend to become extinct

(ii) If $R_{s}>0$, the infected individuals are persistent in mean

(iii) If $R_{s}>0$, the stochastic process $(S(t), I(t), R(t), \xi(t))$ of system (2) is ergodic and has a unique stationary distribution

To verify the correctness of the theoretical analysis, numerical simulation is employed in the following example.

Example 1. In system (2), let $\xi(t)$ be a right-continuous Markov chain taking values in $\mathscr{M}=\{1,2\}$ with the generator 


$$
\Gamma=\left(\begin{array}{cc}
-3 & 3 \\
1 & -1
\end{array}\right)
$$

Then, the unique stationary distribution of $\xi(t)$ is $\pi=\left(\pi_{1}, \pi_{2}\right)=(1 / 4,3 / 4)$. Let $\alpha=0.2$, and other coefficients in system (2) are selected as follows.

For $k=i(i=1,2), \quad(A(1), A(2))=(1.3,1.1), \quad(q(1), q$ $(2))=(0.2,0.3), \quad(\beta(1), \beta(2))=(0.6,0.7), \quad(\mu(1), \mu(2))=$ $(0.45,0.5), \quad(\lambda(1), \lambda(2))=(0.2,0.3), \quad(\omega(1), \omega(2))=(0.3$, $0.2), \quad\left(\sigma_{1}(1), \sigma_{1}(2)\right)=(0.2,0.2), \quad$ and $\quad\left(\sigma_{3}(1), \sigma_{3}(2)\right)=$ $(0.05,0.2)$.

Firstly, let $\left(\sigma_{2}(1) \sigma_{2}(2)\right)=(0.05,0.1)$. This gives $R_{s}=0.52874>0$. According to Theorem 1, the infectious disease is permanent (Figure 1).

Then, let $\left(\sigma_{2}(1), \sigma_{2}(2)\right)=(1.2,1.2)$. This gives $R_{s}=-0.1902<0$. Based on Theorem 1, the disease $I(t)$ goes extinct (Figure 2). It is easy to see that the disease tends to become extinct when the intensity of white noise increases.

Lastly,

$\left(\sigma_{2}(1) \sigma_{2}(2)\right)=(0.05,0.1),(\mu(1), \mu(2))=(0.2,0.15)$. This gives $R_{s}=3.8277>0$. From Theorem 2, the stochastic process $(S(t), I(t), R(t), \xi(t))$ of system (2) is ergodic and has a unique stationary distribution (Figure 3). Figure 3(a) shows the Markov chain, and Figure 3(b) shows a stationary distribution of the stochastic SIRS model. From Figures 3(c)-3(e), we obtain the distributions of $S(t), I(t)$, and $R(t)$ in stochastic system (2), respectively. This means stochastic SIRS model (2) has an ergodic stationary distribution.

\section{Data Availability}

No data were used to support this study.

\section{Conflicts of Interest}

The authors declare that they have no conflicts of interest.

\section{Acknowledgments}

This work was supported by the Shandong Provincial Natural Science Foundation of China (no. ZR2019MA003).

\section{References}

[1] H. W. Hethcote, "The mathematics of infectious diseases," SIAM Review, vol. 42, no. 4, pp. 599-653, 2000.

[2] M. Fan, M. Y. Li, and K. Wang, "Global stability of an SEIS epidemic model with recruitment and a varying total population size," Mathematical Biosciences, vol. 170, no. 2, pp. 199-208, 2001.

[3] J. Li and Z. Ma, "Qualitative analyses of SIS epidemic model with vaccination and varying total population size," Mathematical and Computer Modelling, vol. 35, no. 11-12, pp. 1235-1243, 2002.

[4] Y. Nakata and T. Kuniya, "Global dynamics of a class of SEIRS epidemic models in a periodic environment," Journal of Mathematical Analysis and Applications, vol. 363, no. 1, pp. 230-237, 2010.
[5] V. Capasso and G. Serio, "A generalization of the KermackMcKendrick deterministic epidemic model," Mathematical Biosciences, vol. 42, no. 1-2, pp. 43-61, 1978.

[6] D. Greenhalgh and I. A. Moneim, "SIRS epidemic model and simulations using different types of seasonal contact rate," Systems Analysis Modelling Simulation, vol. 43, no. 5, pp. 573-600, 2003.

[7] C. Ji and D. Jiang, "The threshold of a non-autonomous SIRS epidemic model with stochastic perturbations," Mathematical Methods in the Applied Sciences, vol. 40, no. 5, pp. 1773-1782, 2017.

[8] X. Yu, S. Yuan, and T. Zhang, "Persistence and ergodicity of a stochastic single species model with Allee effect under regime switching," Communications in Nonlinear Science and $\mathrm{Nu}$ merical Simulation, vol. 59, pp. 359-374, 2018.

[9] T. Feng, Z. Qiu, X. Meng, and L. Rong, "Analysis of a stochastic HIV-1 infection model with degenerate diffusion," Applied Mathematics and Computation, vol. 348, pp. 437-455, 2019.

[10] F. Wei and F. Chen, "Stochastic permanence of an SIQS epidemic model with saturated incidence and independent random perturbations," Physica A: Statistical Mechanics and its Applications, vol. 453, pp. 99-107, 2016.

[11] T. Feng, Z. Qiu, and Z. Qiu, "Global analysis of a stochastic TB model with vaccination and treatment," Discrete \& Continuous Dynamical Systems-B, vol. 24, no. 6, pp. 2923-2939, 2019.

[12] Y. Wang, G. Liu, and G. Liu, "Dynamics analysis of a stochastic SIRS epidemic model with nonlinear incidence rate and transfer from infectious to susceptible," Mathematical Biosciences and Engineering, vol. 16, no. 5, pp. 6047-6070, 2019.

[13] G. Lan, Y. Huang, C. Wei, and S. Zhang, "A stochastic SIS epidemic model with saturating contact rate," Physica A: Statistical Mechanics and Its Applications, vol. 529, Article ID 121504, 2019.

[14] W. Guo, Q. Zhang, X. Li, and W. Wang, "Dynamic behavior of a stochastic SIRS epidemic model with media coverage," Mathematical Methods in the Applied Sciences, vol. 41, no. 14, pp. 5506-5525, 2018.

[15] Q. Liu, D. Jiang, D. Jiang, T. Hayat, and A. Alsaedi, "Dynamical behavior of a multigroup SIRS epidemic model with standard incidence rates and Markovian switching," Discrete \& Continuous Dynamical Systems-A, vol. 39, no. 10, pp. 5683-5706, 2019.

[16] Y. Lin and M. Jin, "Ergodicity of a regime-switching epidemic model with degenerate diffusion," Physica A: Statistical Mechanics and Its Applications, vol. 526, Article ID 121134, 2019.

[17] J. Hu, Z. Teng, Z. Li, and B. Wen, "The threshold dynamics in a stochastic SIS epidemic model with vaccination and nonlinear incidence under regime switching," Physica A: Statistical Mechanics and Its Applications, vol. 529, Article ID 121555, 2019.

[18] Q. Liu, D. Jiang, T. Hayat, and B. Ahmad, "Periodic solution and stationary distribution of stochastic SIR epidemic models with higher order perturbation," Physica A: Statistical Mechanics and its Applications, vol. 482, pp. 209-217, 2017.

[19] Y. Zhao and D. Jiang, "The threshold of a stochastic SIS epidemic model with vaccination," Applied Mathematics and Computation, vol. 243, pp. 718-727, 2014.

[20] X. Zhang, D. Jiang, A. Alsaedi, and T. Hayat, "Stationary distribution of stochastic SIS epidemic model with vaccination under regime switching," Applied Mathematics Letters, vol. 59, pp. 87-93, 2016. 
[21] X. Mao and C. Yuan, Stochastic Differential Equations with Markovian Switching, Imperial College Press, London, UK, 2006.

[22] W. Zhao, J. Li, T. Zhang, X. Meng, and T. Zhang, "Persistence and ergodicity of plant disease model with markov conversion and impulsive toxicant input," Communications in Nonlinear Science and Numerical Simulation, vol. 48, pp. 70-84, 2017.

[23] Y. Zhang, K. Fan, S. Gao, and S. Chen, "A remark on stationary distribution of a stochastic SIR epidemic model with double saturated rates," Applied Mathematics Letters, vol. 76, pp. 46-52, 2018. 